\section{Transparency Needed for Economic Analyses of PA Programs}

\section{Dear Editor,}

I am writing in response to the article "Pharmacoeconomic Modeling of Prior-Authorization Intervention for COX-2 Specific Inhibitors in a 3-Tier Copay Plan" in the July/August 2003 issue of the Journal of Managed Care Pharmacy. ${ }^{1}$ While the authors are to be commended for using an economic analysis to assess the impact of a prior-authorization (PA) program, I have some objections with the paper that I wish to express.

My primary objection addresses the lack of transparency in methods used. When conducting pharmacoeconomic analyses, it is essential that the methods be made explicit to the reader. Otherwise, there is no way of knowing of the validity or applicability of the conclusions. In this study, there are several key variables that are insufficiently explained or ignored entirely.

1 . The model assumes a drug cost savings of $\$ 0.31$ per member per month (PMPM) exclusive of the PA program. This savings estimate was calculated based on the difference between the plan's market share of COX-2s to nonspecific NSAIDs with that of other managed care populations across the nation according to IMS Health. The assumption is that the PA plan is responsible for that difference. This is a rather weak assumption upon which to base an entire analysis without additional substantiation. The discrepancy in COX2 use between this plan and that of other plans across the nation could be explained by a variety of alternative causes other than the PA program. The variance could just as easily be explained by differences in population characteristics or variations in practice patterns.

2. The paper fails to mention that a significant portion of the savings resulted from denying care to $13 \%$ of people who have a prescription from their doctor.

3. There was no information provided about how the cost of the PA program was calculated. Readers were told that the PA program cost $\$ 0.07$ per call but were not told whether that sum included costs due to start-up, office space and equipment, employee salaries and benefits, employee training, computing, and administration or how those costs were allocated. At a minimum, $25 \%$ of all costs associated with the PA plan-wide should be allocated toward this program since $25 \%$ of all calls were for COX-2 inhibitors.

4. There was no discussion about the possible relationship between the PA requests for COX-2 inhibitors and PA for proton-pump inhibitors (PPIs). Would a COX-2 PA request to switch to a NSAID and PPI initiate another PA for the PPI? If so, how many PAs for PPIs (20\% of all PAs plan-wide) were generated due to a switch from a COX-2 drug? Were these costs included in the analysis?

5. The study only examined the cost of serious GI events and ignored the costs of other less-serious events. Symptoms of dyspepsia related to the treatment of arthritis have been associated with higher health care resource utilization (e.g., outpatient visits, inpatient admissions). ${ }^{2}$
6. Insufficient details are provided regarding the PA process and its impact on physician, pharmacist, and patient time. How much time was spent by persons affected by the PA burden? For example, how much time did patients, pharmacists, and physicians spend in switching from a prescribed COX-2 to an approved drug? There were no data or discussion relating to these potential time costs.

7. The model does not address costs associated with the requirement that a patient fail on 2 NSAIDs before becoming eligible for a COX-2 drug.

8. Figure 3 suggests that sensitivity analyses were conducted for all probabilities. However, they were not conducted on key assumptions such as the PMPM drug cost, PMPM cost of the PA, risk factors such as age or prior GI event, or percent compliance with medications. In addition, no 2-way or 3-way sensitivity analyses were conducted on any variables, although they are warranted.

9. The limitations section failed to acknowledge many of the study's limitations described above.

My primary concern with this paper is that it presents conclusions with insufficient transparency of methods. Outcomes researchers can make a model agree with any conclusion they desire. Therefore, it is critical that authors make their methods and underlying assumptions as explicit as possible. Casual readers of this paper may accept the conclusions on face value without any understanding of their limitations. Worse, the conclusions may be cited by others to justify similar PA programs.

In conclusion, I want to express a final point relating to the issue of bias or conflict of interest. In the disclosure section at the end of the article, the authors "reported no biases or conflicts of interest in the preparation of this manuscript." This statement lacks credibility given the fact that the authors are all employed by Humana, a health benefits company. Clearly, the authors' interests are advanced if their PA intervention is shown to be cost effective. That does not imply that they are conducting biased research; it only suggests that their source of employment makes it that much more important to be explicit in their methods.

David Holdford, RPh, PhD

Department of Pharmacy, Medical College of Virginia Richmond, Virginia

E-mail: david.holdford@vcu.edu

\section{REFERENCES}

1. Stacy J, Shaw E, Arledge MD, Howell-Smith D. Pharmacoeconomic modeling of prior-authorization intervention for COX-2 specific inhibitors in a 3-tier copay plan. J Managed Care Pharm. 2003;9(4):327-34.

2. Zhao SZ, Argulelles LM, Dedhiya SD, Morgan DG. Healthcare utilization associated with dyspepsia in patients with arthritis. Am J Managed Care. 1999;5(10):1285-95.

For author response, see following page. 


\section{The Authors Respond}

Dr. Holdford raises some good questions regarding our study. ${ }^{1}$ Our point-by-point response is as follows:

1. It is reasonable that population differences or practice variation can contribute to utilization variance. However, the size of the study population does represent broad geographic, demographic, and treatment patterns.

2. There is strong evidence regarding overprescribing of COX-2 specific inhibitors. The number of requests for COX-2 drugs suggests that many prescriptions are written for patients with no risk. The prior-authorization (PA) process is put in place to provide COX-2 drugs based on established risk criteria.

3. The administrative cost per call for the call center in 2000 reflected all costs, including office space and equipment, employee salaries and benefits, training costs, etc. The stated cost savings for the PA program on COX-2 specific inhibitors was adjusted for the entire operation of the call center. If only $25 \%$ of all costs of the call center were used, the cost savings of $\$ 0.24$ per member per month (PMPM) would be more favorable.

4. Concomitant use of proton-pump inhibitors (PPIs) with COX-2 specific inhibitors and nonspecific nonsteroidal antiinflammatory drugs (NSAIDs) was reflected in the population of our model (see Figure 1). The membership is allowed 90-day PPI treatment without a PA. The actual influence of PA rate change for PPIs was not in the scope of the study.

5. This study, along with many in the current literature regarding NSAID treatment, focuses on only serious GI events. The study cited by Holdford used claims data from 1992-1993, before the availability of COX-2 drugs. It would probably be of value to repeat this analysis using more recent claims data.

6. The issues of PA impact on physician and patient time were addressed in the second-to-last paragraph of the Results section. It was acknowledged that the value of PA might decrease due to the level of patient and provider satisfaction and its impact on member retention and burden of use (see bottom of page 332).

7. We agree that the costs associated with treatment failure for nonspecific NSAIDs were not measured in the scope of this study.

8. Sensitivity analysis was conducted on the probabilities in which the assumptions were made. The areas of PMPM drug cost, cost of PA, and risk factors were calculated numbers that we feel were very real estimates with a smaller range of variability. Although this was only a preliminary study, a 2-way sensitivity analysis may prove valuable for future evaluations to determine how one variable affects the other.

Regarding the issue of bias or conflicts of interest, this study was done to help make administrative decisions about benefit design and the true value of PA to the health plan. The results were not biased to sway the outcomes one way or the other.

Jane Stacy, PharmD Clinical Pharmacist, Outcomes Analysis Humana, Louisville, Kentucky E-mail:jstacy1@humana.com

\section{REFERENCE}

1. Stacy J, Shaw E, Arledge MD, Howell-Smith D. Pharmacoeconomic modeling of prior-authorization intervention for COX-2 specific inhibitors in a 3-tier copay plan. J Managed Care Pharm. 2003;9(4):327-34. 